
Revue
de l'histoire
Revue de l'histoire des religions
des religions
2 | 2013
Écrire dans les pratiques rituelles de la Méditerranée antique. Identités et autorités

\title{
Écrire pour Dionysos : la présence de textes écrits dans les rituels dionysiaques
}

Writing for Dionysus: the presence of written texts in the Dionysiac rituals

\section{Francesco Massa}

\section{OpenEdition}

\section{Journals}

Édition électronique

URL : http://journals.openedition.org/rhr/8111

DOI : $10.4000 /$ rhr.8111

ISSN : 2105-2573

Éditeur

Armand Colin

Édition imprimée

Date de publication : 1 juin 2013

Pagination : 209-232

ISBN : 978-2-200-92864-3

ISSN : 0035-1423

Référence électronique

Francesco Massa, "Écrire pour Dionysos : la présence de textes écrits dans les rituels dionysiaques », Revue de l'histoire des religions [En ligne], 2 | 2013, mis en ligne le 01 juin 2016, consulté le 30 avril 2019. URL : http://journals.openedition.org/rhr/8111 ; DOI : 10.4000/rhr.8111 


\section{Écrire pour Dionysos : la présence de textes écrits dans les rituels dionysiaques}

L'article se propose d'analyser les traces de l'écriture dans la ritualité bachique au travers de diverses séries de documents (textes, inscriptions, images). L'étude pose d'autre part des questions sur le contenu de ces écrits: abordaient-ils des problèmes rituels et évoquaient-ils des récits mythiques? Étaient-ils conçus comme des supports rituels, comme des répertoires de formules ou de récits, ou bien encore comme des commentaires aux pratiques et aux récits?

\section{Writing for Dionysus: the presence of written texts in the Dionysiac} rituals.

This paper tackles the presence of written texts within Dionysiac rituality through the examination of various kinds of evidence (texts, engravings, images). The study then addresses a few questions regarding the contents of these written texts: are these texts concerned with ritual problems or mythical narratives? Were they designed to be used as props for rituals, as repertories of formulas or narratives or, rather, as commentaries of Dionysiac practices and narratives? 


\section{Cultes dionysiaques eT PRATIQUes d'ÉCRITURES : AU-DELÀ DE L'ORPHISME}

Au retour d'un voyage qui l'a éloigné d'Athènes, le roi Thésée arrive devant son palais et découvre que Phèdre, son épouse, s'est donné la mort en se pendant dans sa chambre. Elle tient encore à la main une tablette dans laquelle elle explique les raisons de son geste et accuse Hippolyte, son beau-fils, d'avoir cherché à la violenter. Selon l'intrigue créée par Euripide, avant que l'accusation de Phèdre ne s'effondre, Thésée affronte son fils en lui reprochant sa conduite et ses croyances religieuses :

C'est toi l'homme supérieur qui vit dans la société des dieux? toi, l'être vertueux, pur de tout mal? Non, tes vantardises ne me persuaderont pas d'être assez fou pour taxer les dieux d'esprits faux. Va maintenant, glorifie-toi; avec ton régime végétarien fais étalage de ta nourriture; sous la direction d'Orphée, joue l'inspiré, tiens en

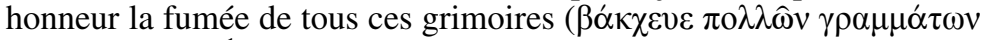
$\tau \mu \hat{\omega} \nu \kappa \alpha \pi \nu 0 u ́ \varsigma)^{1}$.

Hippolyte, dans les veines duquel coule le sang d'Antiope, reine des Amazones, représente une conception religieuse qui s'éloigne des règles de la polis: des éléments dionysiaques, pythagoriciens et «orphiques» se mêlent dans la figure littéraire euripidéenne et créent l'image d'un dévot de cultes non traditionnels, qui fait de la chasteté et du régime alimentaire végétarien deux principes fondamentaux ${ }^{2}$.

1. Euripide, Hippolyte 948-954: traduction de Louis Méridier et François Jouan, dans Euripide, Tragédies, tome II Hippolyte, Andromaque, Hécube, Paris, 1997, p. 65-66.

2. Marcel Detienne a parlé de «chemins de déviance» pour réunir la conduite des dévots d'Orphée, Dionysos et Pythagore, qui, malgré les formes spécifiques de chaque forme cultuelle, seraient caractérisés par le refus des traditions rituelles de la polis de l'âge classique: cf. «Le chemin de la déviance: orphisme, dionysisme, pythagorisme», dans Orfismo in Magna Grecia. Atti del quattordicesimo Convegno di Studi sulla Magna Grecia (Taranto, 6-10 ottobre 1974), Napoli, 1975, p. 49-79 et «Pratiques culinaires et esprit de sacrifice», dans Marcel Detienne, Jean-Pierre Vernant éds., La cuisine du sacrifice en pays grec, Paris, 1979, p. 7-35. Sur le passage de l'Hippolyte d'Euripide, voir aussi Ruth Scodel, «Euripides, the Derveni Papyrus, and the Smoke of Many Writings », dans André Lardinois, Josine Blok, Marc van der Poel éds., Sacred Words : Orality, Literacy and Religion (Mnemosyne Supplements, 332), Leiden-Boston, 2011, p. 79-98. 
La représentation de l'Hippolyte d'Euripide à travers le discours de Thésée est un bon point de départ pour l'analyse du rôle de l'écriture dans les pratiques rituelles dionysiaques: dans le texte tragique, en effet, le verbe $\beta \alpha \kappa \chi \varepsilon v ́ \omega$ est directement lié au fait de vénérer la «fumée de beaucoup d'écrits». Pourtant, loin d'être évidente, la présence ou l'utilisation de textes écrits dans les cultes dionysiaques ne se laisse appréhender que par le croisement et la comparaison de différentes sources : textes littéraires, inscriptions et images. Cette étude part donc à la recherche des traces de l'écriture dans la ritualité bachique dans les diverses séries de documents dont nous disposons, et propose une mise au point méthodologique des possibilités d'exploitation du matériel sélectionné en fonction du type de document. Quant au sens et au contenu de ces écrits, nous poserons les questions qu'ils soulèvent, même si les réponses restent, en l'état actuel de la documentation, bien évanescentes: ces écrits abordaient-ils des questions rituelles et/ou des récits mythiques? Étaient-ils conçus comme des supports rituels, des répertoires de formules ou de récits, ou bien encore comme des commentaires aux pratiques et aux récits? Telles sont les problématiques qui guident cette étude des différents vestiges potentiels de l'usage de l'écrit dans la ritualité dionysiaque.

Dans la civilisation grecque de la deuxième moitié du $\mathrm{v}^{\mathrm{e}}$ siècle avant notre ère - où l'écriture est en train de conquérir ses espaces d'autonomie dans des domaines intellectuels (comme la médecine, l'histoire, la géographie, la géométrie) -, l'oralité demeure la modalité de transmission et de déroulement des cérémonies religieuses ${ }^{3}$, même si les cultes de la polis utilisent l'écriture comme support des pratiques rituelles, certes de façon encore limitée. Le fonds documentaire de l'époque classique ne laisse affleurer que d'infimes traces de l'utilisation de textes écrits dans certains milieux religieux. Pour ce qui concerne les pratiques cultuelles dionysiaques, deux exemples liés à des pratiques initiatiques confirment la présence de l'écriture, du moins au $\mathrm{IV}^{\mathrm{e}}$ siècle avant notre ère. Dans son discours Sur la couronne (en 330), l'orateur

3. Cf. Albert Henrichs, «Writing Religion: Inscribed Texts, Ritual Authority and the Religious Discourse of the Polis », dans Harvey Yunis éd., Written Texts and the Rise of Literate Culture in Ancient Greece, Cambridge, 2003, p. 38-58. Voir également André Lardinois, Josine Blok, Marc van der Poel éds., Sacred Words: Orality, Literacy and Religion. 
attique Démosthène présente à son public une image délibérément ridicule de son adversaire politique Eschine, occupé à la pratique de cultes initiatiques liés à la fois à Dionysos (la présence de thiases et de nébrides) et à Sabazios (l'invocation «Evohé! Sabohé !»). La procédure de ces initiations comporte la lecture de livres qui devaient probablement renfermer diverses prescriptions pour l'accomplissement des rituels :

Devenu homme, pendant que ta mère pratiquait ses initiations, tu

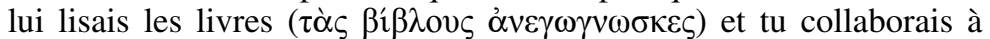
toutes ses machinations: toute la nuit, tu présentais la peau de faon et le cratère, tu purifiais les initiés, tu les barbouillais de boue et de son, tu les faisais lever après l'initiation, tu leur faisais dire: «J'ai échappé au mal, j'ai trouvé le bien », tout fier que personne n'eût jamais poussé de tels hurlements ${ }^{4}$.

D'après Démosthène, des textes écrits - de véritables $\beta$ í $\beta \lambda o r$ et plus seulement des $\gamma \rho \alpha ́ \mu \mu \alpha \tau \alpha$ comme chez Euripide - interviennent dans la célébration de rituels initiatiques dionysiaques. Pourtant, pour éviter de surinterpréter ce passage, il faut tenir compte de deux considérations. En premier lieu, il ne faut pas sous-estimer le ton ironique employé par Démosthène pour décrire les pratiques féminines qui avaient lieu dans la maison d'Eschine: le récit tourne en ridicule son adversaire politique par l'exposé de ses pratiques rituelles, comme il le fait d'habitude par le biais de ses origines modestes et de ses métiers dégradants. On ne saurait occulter la volonté caricaturale de Démosthène lorsqu'il évoque les livres. L'existence d'une tonalité négative dans l'utilisation de textes écrits pourrait être confirmée par un passage de la République de Platon, qui associe la «masse de livres » ( $\beta$ í $\beta \lambda \omega v$ ö $\mu \alpha \delta o v)$ de Musée et d'Orphée à la critique de l'orphisme, lorsque Glaucon dénonce

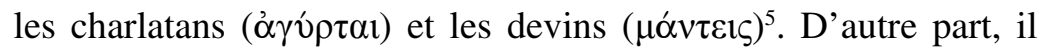
est impossible d'identifier une pratique initiatique unique, qui correspondrait à la représentation que livre l'orateur attique, même si la composante dionysiaque est prépondérante. Ici, comme chez Euripide, c'est moins la description d'une forme rituelle spécifique

4. Démosthène, Discours XVIII, 259: traduction de Georges Mathieu dans Démosthène, Plaidoyers politiques, tome IV, Paris, 1947, p. 107.

5. Cf. Platon, République II, 364c-365a. Voir Claude Calame, «Pratiche orfiche della scrittura: itinerari iniziatici?», dans Giulio Guidorizzi, Marxiano Melotti éds., Orfeo e le sue metamorfosi. Mito, arte, poesia, Roma, 2005, p. 28-45. 
que l'image générale de cultes étrangers et secrets que les deux auteurs ont voulu souligner dans un but polémique.

Si l'on sort des contextes potentiellement polémiques évoqués jusqu'ici, le lien entre les formes de ritualité dionysiaque et l'usage de l'écriture semble confirmé au siècle suivant dans deux lamelles d'or en forme de feuilles de lierre retrouvées à Pélinna, en Thessalie. Elles datent de la fin du $\mathrm{IV}^{\mathrm{e}}$ siècle avant notre ère ${ }^{6}$ :

Tour à tour tu es mort et tu es né, ô trois fois bienheureux, en ce jour.

Dis à Perséphone que c'est Bacchios lui-même qui t'as délivré

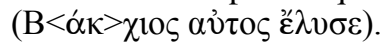

Taureau, tu as couru vers le lait;

immédiatement vers le lait tu as couru;

agneau, tu t'es élancé vers le lait.

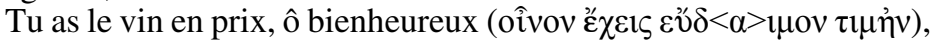

et sous terre t'attendent les rites sacrés que les autres bienheureux célèbrent ${ }^{7}$.

Le caractère dionysiaque de la lamelle est évident, à lire les évocations de Dionysos (dans sa fonction de libérateur, $\beta \alpha ́ \alpha \chi 10 \varsigma$ ) et du vin, ainsi que la forme en feuille de lierre du support. Le texte s'adresse à la défunte et conserve la formule qui permet d'avoir accès au bonheur après la mort: la lamelle est interprétée comme une sorte de talisman d'un initié du dieu Dionysos ${ }^{8}$. Dans ce cadre, l'écriture est fonctionnelle: elle est liée à la fixation du «mot de passe» nécessaire pour se présenter devant la reine des Enfers, Perséphone. À la suite de Claude Calame ${ }^{9}$, on en vient même à se

6. Le texte des deux lamelles est proche, mais l'une conserve deux vers en plus. Les lamelles d'or, qu'on appelle désormais «orphiques» par convention, ont été au centre de débats au cours des dernières années: pour ne citer que les dernières publications, voir Alberto Bernabé, Ana I. Jiménez San Cristóbal éds., Instructions for the Netherworld: The Orphic Gold Tablets, Leiden-Boston, 2007; Fritz Graf, Sarah Iles Johnston, Ritual Texts for Afterlife. Orpheus and the Bacchic Gold Tablets, London-New York, 2007 et Radcliffe G. Edmonds éd., The Orphic Gold Tablets and Greek Religion: Further Along the Path, Cambridge, 2011.

7. Orphica 485 F Bernabé. Traduction française de Giovanni Pugliese Carratelli, Les lamelles d'or orphiques: instructions pour le voyage d'outre-tombe des initiés grecs, Paris, 2003, p. 118. Pour un commentaire des deux lamelles, Fritz Graf, «Textes orphiques et rituel bacchique. À propos des lamelles de Pélinna», dans Philippe Borgeaud éd., Orphisme et Orphée en l'honneur de Jean Rudhardt, Genève, 1991, p. 87-102.

8. Voir Fritz Graf, «Textes orphiques», p. 90.

9. Sur le caractère performatif du texte, Claude Calame, «Funerary Gold Lamellae and Orphic Papyrus Commentaries: Same Use, Different Purpose», dans 
demander dans quelle mesure le texte écrit n'est pas partie intégrante du rite par sa valeur performative. Lu une première fois lors du rituel initiatique, le texte est censé développer tout son potentiel d'efficience rituelle lorsqu'il sera lu ou déclamé par le défunt face aux puissances rencontrées dans le voyage vers l'Hadès. Dans cette perspective, l'écrit prend une dimension centrale dans le rite.

L'écriture apparaît de toute évidence comme un instrument, parfois même central, utilisé lors de rituels initiatiques bachiques. Mais le passage de l'Hippolyte, comme les lamelles d'or de Pélinna, sont également importants au regard du débat critique qu'ils ont généré: leur inscription dans le cercle des sources «orphiques» est à l'origine de l'hypothèse d'une possible coïncidence et superposition entre les formes cultuelles de l'«orphisme» et du «dionysisme $»^{10}$. De la bibliographie la plus récente sur la question ressortent immanquablement deux thèses implicites et interdépendantes: d'une part l'«orphisme» absorberait les traits spécifiques du culte de Dionysos et en proposerait une sorte de version supérieure, philosophique et, d'autre part, les textes écrits ne sauraient s'associer qu' au sérieux et à la profondeur mystique du premier, ne laissant au second que l'action, notamment la transe. En d'autres termes, il existerait, dans les cérémonies initiatiques dionysiaques, une partie proprement «orphique» - représentée par les hieroi logoi du chantre thrace Orphée - et une partie «dionysiaque» constituée par les pratiques orgiastiques ${ }^{11}$. Pourtant, si, dans l'Hippolyte d'Euripide, le nom d'Orphée est directement associé au verbe $\beta \alpha \kappa \chi \varepsilon v ́ \omega$, chez Démosthène et dans les lamelles d'or de Pélinna, il n'est pas question de pratiques ou croyances orphiques, malgré l'utilisation manifeste de textes écrits dans le rituel $^{12}$.

Radcliffe G. Edmonds éd., The Orphic Gold Tablets and Greek Religion, p. 203218.

10. Ainsi par exemple Ana I. Jiménez San Cristóbal, «Orfismo y dionisismo », dans Alberto Bernabé, Francesc Casadesús éds., Orfeo y la tradición órfica. Un reencuentro, Madrid, 2008, p. 697-727.

11. Voir par exemple Gabriella Ricciardelli, «L'elemento spettacolare nel rito orfico-dionisiaco », La Parola del Passato 376, 2010, p. 98-126.

12. Contre la prétendue nature orphique des lamelles de Pélinna, Claude Calame, «Qu'est-ce qui est orphique dans les Orphika?», Revue de l'Histoire des Religions 219, 4, 2002, p. 385-400, en particulier p. 390-391. Voir aussi Id., «Les lamelles funéraires d'or: textes pseudo-orphiques et pratiques rituelles? », Kernos 21, 2008, p. 299-311. 
La question des rapports entre les catégories modernes de l'«orphisme» et du «dionysisme» est particulièrement complexe. Dès l'époque classique certes, Orphée était reconnu comme l'auteur de poèmes et textes sacrés portant parfois le nom de hieroi logoi, et, à partir de l'époque impériale, des auteurs comme Plutarque et Lucien associent cette expression au chantre thrace ${ }^{13}$. Mais le nom d'Orphée n'est associé de manière explicite aux cultes dionysiaques qu'à partir du $\mathrm{I}^{\mathrm{er}}$ siècle avant notre ère ${ }^{14}$; et seuls les fragments des Discours en 24 rhapsodies (fin du I $^{\mathrm{er}}$-début du II $^{\mathrm{e}}$ siècle de notre ère), transmis par Damascius et Proclus, situent le mythe de Dionysos et les Titans sous le signe d'Orphée ${ }^{15}$. C'est pourquoi la présente étude se propose d'éclairer la fonction de l'écriture exclusivement dans les pratiques dionysiaques, par delà les contaminations possibles avec l'ensemble des croyances associées à la figure d'Orphée. Nous analyserons notamment les rites initiatiques bachiques désignés par les termes grecs teletai, musteria ou orgia.

\section{DES SABLES D'ÉGYPTE : PAPYRUS ET HIEROI LOGOI}

Deux papyrus égyptiens permettent d'aborder la question du contenu des textes utilisés dans les teletai dionysiaques. Le premier est un document relevant de l'administration du royaume des Ptolémées, alors que le deuxième est un texte fragmentaire complexe à définir, qui évoque le cadre d'un rituel initiatique.

Un papyrus retrouvé en Égypte conserve un décret royal ptolémaïque ordonnant aux initiés de Dionysos de «descendre» jusqu'à Alexandrie pour remettre une copie de leur hieros logos:

Par ordre du roi, ceux qui dans le pays initient aux cultes de

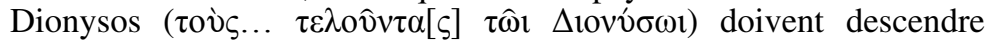
(le fleuve) jusqu'à Alexandrie; ceux qui n'habitent pas au-delà de Naucratis dans 1'espace de dix jours de la proclamation du présent édit, ceux qui habitent au-delà de Naucratis dans l'espace de vingt jours, et ils devront s'inscrire par- devant Aristobule au bureau

13. Cf. Plutarque, Propos de table II, 636d et Lucien, De l'astrologie X. À ce propos, Albert Henrichs, "Hieroi Logoi and Hierai Bibloi: The (Un)Written Margins of the Sacred in Ancient Greece », Harvard Studies in Classical Philology 101, 2003, p. 207-266, en particulier p. 214-215.

14. Cf. Cicéron, De natura deorum III, 58.

15. Voir Luc Brisson, Orphée et l'orphisme dans l'antiquité gréco-romaine, Paris, 1995, p. 5. 


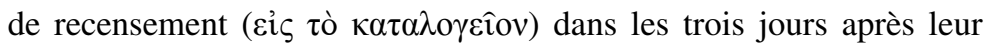
arrivée, déclarer immédiatement de qui ils ont reçu les rites ( $\tau \dot{\alpha}$ i $\varepsilon \rho \alpha ́$ )

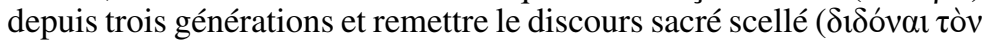

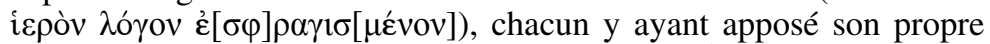

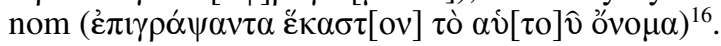

Le texte du papyrus impose aux initiateurs des cultes de

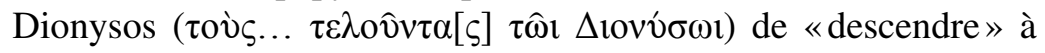
Alexandrie, de s'inscrire au bureau de recensement, de déclarer l'origine des rites sacrés et de remettre la copie scellée du hieros logos. La datation du décret est située sous le règne de Ptolémée IV Philopator (221-204 avant notre ère), dont on connaît la dévotion et l'intérêt pour le dieu Dionysos ${ }^{17}$. La nature et les buts de ce texte de l'administration ptolémaïque ont été au centre d'un débat qui a suscité diverses hypothèses: un acte préparatoire à la suppression des cultes initiatiques dionysiaques en Égypte - à l'instar de ce qui se passera bientôt à Rome en 186 -, ou la volonté de coordonner et d'unifier les diverses traditions mythiques et rituelles dionysiaques, ou encore simplement une mesure fiscale destinée à la perception d'une contribution pour les associations dionysiaques ${ }^{18}$, nous y reviendrons.

L'interprétation de l'édit dépend en partie de la valeur que l'on attribue à l'expression hieros logos et à son contenu: il n'est pas facile de comprendre si elle se réfère à un récit mythique, à une série d'actions rituelles ou bien à un texte à la fois mythique et rituel $^{19}$. Si le hieros logos contenait également un certain nombre de prescriptions sacrées, il se rapprocherait de ce qu' on intitule hieros nomos. Dans le papyrus égyptien, il est question d'un logos, transmis

16. C. Ord. Ptol. $29=B G U$ VI 1211 ; traduction Furio Jesi, «Notes sur l'édit dionysiaque de Ptolémée IV Philopator», Journal of Near Eastern Studies 15, 4, 1956, p. 236-240 (p. 236).

17. Sur les témoignages archéologiques et littéraires concernant le lien de Ptolémée IV avec le dieu Dionysos, Françoise Dunand, "Les associations dionysiaques au service du pouvoir lagide (III ${ }^{\mathrm{e}} \mathrm{s}$. av. J.-C.)», dans L'association dionysiaque dans les sociétés anciennes (Actes de la table ronde organisée par l'École française de Rome, 24-25 mai 1984), Roma, 1986, p. 85-104, en particulier p. 87-88.

18. Sur ces interpretations, Günther Zuntz, «Once More: the So-Called Edict of Philopator on the Dionysiac Mysteries», Hermes 91, 1963, p. 228-239.

19. Pour une réflexion générale sur la nature des hieroi logoi, Albert Henrichs, «Hieroi Logoi», p. 231-250, Roland Baumgarten, Heiliges Wort und Heilige Schrift bei den Griechen: Hieroi Logoi und verwandte Erscheinungen, Tübingen, 1998 et Fritz Graf, Sarah Iles Johnston, Ritual Texts, p. 175-184. 
de génération en génération et utilisé par ceux qui accomplissent les initiations dionysiaques. Mais l'édit ne permet pas d'expliciter les modalités d'une telle transmission: faut-il imaginer un discours oral ou un discours écrit avant l'enregistrement à Alexandrie ? Nous pouvons seulement être certains de l'existence d'une version écrite sur laquelle devait être apposé un sceau du bureau ptolémaïque: s'agit-il d'un texte rédigé par les initiateurs aux cultes de Dionysos ou par le préposé administratif? La question reste ouverte.

Les autres attestations de l'expression hieros logos sont liées aux pratiques des cérémonies mystériques et font référence à une explication du contenu d'un rituel ${ }^{20}$. Hérodote emploie l'expression à plusieurs reprises dans son deuxième livre consacré à l'Égypte, autant pour définir des logoi égyptiens que des logoi dévoilés pendant des cérémonies du monde grec ${ }^{21}$. Pausanias lui-même utilise l'expression au singulier pour indiquer un récit sacré concernant l'interdiction de se nourrir de fèves, pratique répandue chez les Phénéates dans l'initiation ( $\tau \varepsilon \lambda \varepsilon \tau \eta \hat{)})$ de Déméter ${ }^{22}$. Hérodote comme Pausanias se réfèrent vraisemblablement à des discours oraux, des récits «sacrés » dont il faut garder le secret en raison de leur contenu lié aux pratiques rituelles initiatiques. Les sources anciennes ne donnant pas d'indication sur la nature écrite ou orale des hieroi logoi, certains savants ont supposé qu'il s'agissait de discours qui circulaient sous une forme écrite garantissant la préservation de leur contenu dans la mesure où ils étaient utilisés par des petits groupes $^{23}$. Cependant cette hypothèse ne correspond pas à ce que les sources disent à propos des hieroi logoi ${ }^{24}$. L'édit de Ptolémée ne suppose pas que le hieros logos dionysiaque soit, depuis son origine, un texte écrit ${ }^{25}$; la remise d'une copie scellée n'implique pas que, à l'annonce du décret, dans tous les nomes d'Égypte, les prêtres dionysiaques aient déjà écrit leurs discours sacrés pour

20. Cf. Fritz Graf, Sarah Iles Johnston, Ritual Texts, p. 176.

21. Cf. Hérodote II, 48,3 (cultes dionysiaques); 51,4 (mystères de Samothrace); 62, 2 (fête égyptienne); 81, 2 (interdiction de l'usage de la laine). Sur l'emploi hérodotéen de l'expression, Albert Henrichs, «Hieroi Logoi», p. 235-239.

22. Pausanias VIII, 15, 4. Cf. aussi Plutarque, Isis et Osiris 353d.

23. Cf. Fritz Graf, Sarah Iles Johnston, Ritual Texts, p. 180-181.

24. Cf. Albert Henrichs, «Hieroi Logoi», p. 240: «In Greece proper, hieroi logoi remained by definition unwritten».

25. Contra Walter Burkert, Ancient Mystery Cults, Cambridge [Mass.], 1987, p. 33: «Part of the tradition is a "sacred tale" in written form». 
accomplir les pratiques cultuelles. La tendance à la mise par écrit dérive d'abord de la volonté de la bureaucratie ptolémaïque, à en juger par l'enregistrement auprès des archives publiques. Toutefois, on ne peut exclure une circulation de hieroi logoi écrits, utiles aux pratiques initiatiques, comme le suggère le Papyrus de Gurôb, ainsi que nous le verrons.

La mise parécrit du contenu du hieros logos permet de réfléchir aux objectifs et aux fonctions mêmes de l'écriture. Face à l'importance du culte de Dionysos dans l'Égypte ptolémaïque, et notamment à Alexandrie ${ }^{26}$, un décret destiné à la suppression des cérémonies dionysiaques paraît difficilement envisageable. Il est plus probable d'envisager une volonté de la maison royale de recenser les groupes bachiques présents dans les territoires égyptiens, afin d'en comprendre la nature et les pratiques rituelles, du fait de l'intérêt que la dynastie des Ptolémées montrait à l'égard du dieu Dionysos. L'écriture et l'enregistrement semblent donc se présenter comme un instrument de contrôle des pratiques dionysiaques et des prêtres bachiques chargés d'accomplir les initiations. Même si le papyrus de Ptolémée IV a été utilisé parfois comme un document permettant d'illustrer la structure générale des organisations initiatiques dionysiaques à l'époque hellénistique (présence de prêtres itinérants, utilisation de hieroi logoi dans les pratiques rituelles, etc. $)^{27}$, le décret demeure le résultat d'une réalité égyptienne propre, qui n'explique pas nécessairement l'organisation interne de toutes les associations bachiques des mondes grec et romain.

À côté de l'édit de Ptolémée, la découverte d'un deuxième papyrus, connu sous le nom de Papyrus de Gurôb ${ }^{28}$, vient confirmer la circulation de textes écrits à contenu dionysiaque en Égypte. Le texte, de forme

26. Pour une présentation du rôle des associations dionysiaques dans l'Égypte $\mathrm{du} \mathrm{III}^{\mathrm{e}}$ siècle avant notre ère, Françoise Dunand, «Les associations dionysiaques » et Walter Burkert, «Bacchic Teletai in the Hellenistic Age», dans Thomas H. Carpenter, Christopher A. Faraone éds., Masks of Dionysus, Ithaca-London, 1993, p. 259-274, en particulier p. 262-264.

27. Ainsi par exemple, Walter Burkert, Ancient Mystery Cults, p. 33.

28. Le Papyrus de Gurôb se compose de deux colonnes dont on ne peut lire que des parties. La première a été publiée en 1921 et les deux dans le recueil d'Otto Kern, Orphicorum fragmenta, Berlin, 1922, fr. 31. Voir aussi Alberto Bernabé, Poetae epici Graeci, vol. II Orphicorum Graecorum testimonia et fragmenta, München, 2004, fr. 578 F. Pour l'édition la plus récente du texte, James Hordern, «Notes on the Orphic Papyrus from Gurôb (P. Gurôb 1; Pack $\left.{ }^{2} 2464\right)$ ), Zeitschrift für Papyrologie und Epigraphik 129, 2000, p. 131-140. 
poétique (peut-être hexamétrique), date de la deuxième moitié du III $^{\mathrm{e}}$ siècle avant notre ère: bien qu'extrêmement fragmentaire, il est possible d'y lire une série d'indications à suivre dans la célébration d'un rituel, puisqu'il y est explicitement fait mention d'une teletè. Le lieu de la découverte, la région égyptienne du Fayoum, a conduit certains savants à lire le papyrus comme l'un des hieroi logoi dionysiaques dont il est question dans l'édit de Ptolémée ${ }^{29}$.

Plusieurs chercheurs ont interprété le document comme un témoignage «orphique» ${ }^{30}$ - sachant que l'expression «orphicodionysiaque » est généralement utilisée pour désigner un amalgame d'éléments communs aux deux formes religieuses. Selon James Hordern, le dernier éditeur du texte, le papyrus contiendrait une série d' «instructions for a ritual based around the death (and rebirth?) of the infant Dionysus», c'est-à-dire pour le mythe de Dionysos et les Titans ${ }^{31}$. Pourtant le texte ne fait pas explicitement référence à cet épisode de la vie de Dionysos, et encore moins à sa mort: comme l'ont déjà souligné Luc Brisson et Claude Calame, le papyrus de Gurôb appartient plutôt aux traditions dionysiaque et éleusinienne ${ }^{32}$.

L'état lacunaire du papyrus ne permet pas de proposer une interprétation globale du texte. Cependant, il fait référence à une initiation ( $\tau \varepsilon \lambda \varepsilon \tau \eta \dot{v} v$, ligne 3 ) et, sans doute, à la prescription rituelle de ramasser les pièces de viande crue, conformément au rite du sparagmos dionysiaque (ligne 2), qui pourrait se référer au bélier et au bouc évoqués dans les lignes suivantes. Suit l'injonction pour les non-initiés de ne pas assister aux cérémonies (ligne 16), et une série de noms de divinités - parmi lesquels Brimò, Déméter, Rhéa, les Curètes, Pallas et Erikêpaios (le seul qui pourrait renvoyer aux théologies orphiques). La dimension bachique ressort des figures

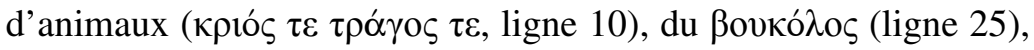

29. Cf. Henri Jeanmaire, Dionysos. Histoire du culte de Bacchus, Paris, 1951, p. 383 et Walter Burkert, Ancient Mystery Cults, p. 70-71.

30. Cf. Martin L. West, The Orphic Poems, Oxford, 1983, p. 170-171 et Marisa Tortorelli Ghidini, Figli della terra e del cielo stellato, Napoli, 2006, p. 255-277. Cf. aussi Paolo Scarpi, Le religioni dei misteri, Milano, 2003, I, p. 654-657, qui situe, avec prudence, le Papyrus de Gurôb dans la section orphique de son recueil de textes.

31. James Hordern, «Notes on the Orphic Papyrus from Gurôb», p. 131.

32. Cf. Luc Brisson, Orphée et l'orphisme, p. 9, qui met en évidence la présence d'éléments éleusiniens qui rendent «problématique son identité orphique» et Claude Calame, «Qu'est-ce qui est orphique dans les Orphika?», p. 391-392. 
ainsi que de la pratique du démembrement des chairs, de la présence

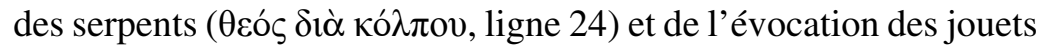
qui seraient à interpréter comme les symbola des «mystères de Dionysos » selon Clément d'Alexandrie ${ }^{33}$.

Le rôle de l'écriture dans ce texte est plus concret: il devient probablement un présupposé nécessaire à la célébration de la pratique rituelle. Certes, la nature fragmentaire du papyrus empêche une compréhension globale et il serait dangereux de vouloir toujours lire des formules, des symbola mystériques, dans des expressions qui pourraient assumer un sens différent si l'on connaissait le contexte dans lequel elles s'inséraient ${ }^{34}$. L'hypothèse selon laquelle le Papyrus de Gurôb contiendrait le texte d'un des hieroi logoi cités dans l'édit de Ptolémée reste à confirmer, même si les coïncidences chronologique et géographique nous invitent à réfléchir sur l'utilisation de l'écriture dans le cadre des initiations dionysiaques de l'Égypte à l'époque hellénistique.

\section{LES FONCTIONS DES INITIÉS DANS LES ASSOCIATIONS DIONYSIAQUES}

Pour dépasser le cadre égyptien et se tourner vers d'autres territoires des mondes grec et romain, il faut analyser non plus les sources littéraires, mais les sources épigraphiques et les documents figurés. Un premier terrain d'investigation s'offre avec les noms des fonctions assumées par les membres des associations dionysiaques.

Une inscription d'Éphèse datée de l'époque d'Hadrien, qui provient d'un revêtement mural qui faisait partie du théâtre de la cité, renvoie à la question des discours sacrés, car elle conserve l'attestation de

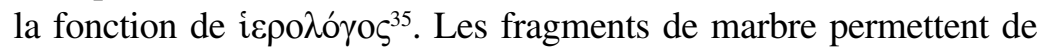
reconstruire notamment une liste d'hommes qui ont participé aux

33. Cf. Clément d'Alexandrie, Protreptique aux Hellènes II, 17, 2. Sur les jouets du Dionysos enfant, Marisa Tortorelli Ghidini, Figli della terra, p. 272275 et Olga Levaniouk, «The Toys of Dionysus», Harvard Studies in Classical Philology 103, 2007, p. 165-202.

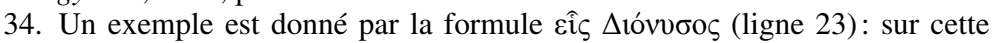
expression, Luc Brisson, Orphée et l'orphisme, p. 2928-2929. Plus généralement sur ce type de formules d'acclamation, Nicole Belayche, «Deus deum... summorum maximus (Apuleius): Ritual Expressions of Distinction in the Divine World in the Imperial Period», dans Stephen Mitchell, Peter van Nuffelen éds., One God. Pagan Monotheism in the Roman Empire, Cambridge, 2010, p. 141-166.

35. IK 15, 1601; cf. le commentaire d'Anne-Françoise Jaccottet, Choisir 
sacrifices dionysiaques. Dans la série, à côté du «prêtre (i $\varepsilon \rho \varepsilon[\hat{c} \varsigma])$ » et $\mathrm{du}$ «porteur du thyrse ( $\theta v] \rho \sigma o \varphi o ́ \rho o \varsigma) »$ apparaît également un

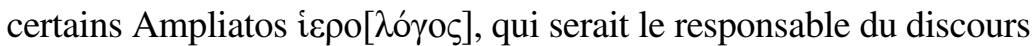
ou du texte sacré. L'état fragmentaire de l'inscription empêche de se prononcer sur la nature de ce groupe dionysiaque : le titre d' «intronisé ('́v $\theta \rho o ́ v ı[\varsigma]) \gg$ pourrait évoquer une intronisation relevant d'une forme d'initiation, mais, comme le relève Anne-Françoise Jaccottet, «les éléments manquent pour une interprétation définitive ${ }^{36}$. En tant que fonction dionysiaque, et plus généralement titre dans les associations initiatiques, le hierologos représente un hapax. Pourtant sa présence à l'intérieur du groupe permet de penser qu'il était chargé de la conservation d'un texte sacré et/ou de sa lecture ${ }^{37}$.

Dans le même ordre d'idées, on peut avancer la fameuse inscription bachique de Torre Nova (trouvée dans le Latium et conservée au Metropolitan Museum de New York), qui date de la moitié du $\mathrm{II}^{\mathrm{e}}$ siècle de notre ère ${ }^{38}$. Cette base en marbre, qui porte une inscription en grec, est dédiée à la prêtresse (ićpєı $\alpha$ ) Agrippinilla par des initiés ( $\mu$ v́ $\tau \alpha$ ). Dans la longue liste, nous lisons vingt-six fonctions d'hommes et de femmes de l'association correspondant au thiase des familiae des Pompeii et des Gavii: la hiérarchie des fonctions dionysiaques est structurée selon l'importance sociale des composants de la famille. Parmi ces titres, on lit pour un certain

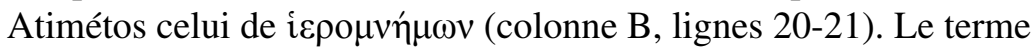
se réfère, en général, à la fonction de secrétaire, d'archiviste ${ }^{39}$, ou bien à celle de pontifex dans le milieu romain ${ }^{40}$. Pourtant, dans cet

Dionysos. Les associations dionysiaques ou la face cachée du dionysisme, Zürich, 2003, II, n ${ }^{\circ} 137$ p. 233-235.

36. Anne-Françoise Jaccottet, Choisir Dionysos, vol. II, p. 234.

37. Robert Turcan parle d'un «lecteur du texte sacré»: Liturgies de l'initiation bacchique à l'époque romaine (LIBER). Documentation littéraire, inscrite et figurée, Paris, 2003, p. 69.

38. IGVR 160. La bibliographie sur cette inscription est particulièrement fournie depuis Franz Cumont, «La grande inscription bachique du Metropolitan Museum. II. Commentaire religieux de l'inscription», American Journal of Archaeology 37, 2, 1933, p. 232-263: cf. Martin P. Nilsson, The Dionysiac Mysteries in the Hellenistic and Roman Age, Lund, 1957, p. 51-52, Henri Jeanmaire, Dionysos, p. 470-471, John Scheid, «Le thiase du Metropolitan Museum (IGVR I 160)», dans L'association dionysiaque, p. 275-290 et Anne-Françoise Jaccottet, Choisir Dionysos, I, p. 30-53 et II, p. 302-310 ( $\left.{ }^{\circ} 188\right)$.

39. Cf. Aristote, Politique 1321 b39. Sur ce titre, Franz Cumont, La grande inscription bachique, p. 254.

40. Cf. Dion Cassius VIII, 55, 3 et Strabon V, 3, 2. 


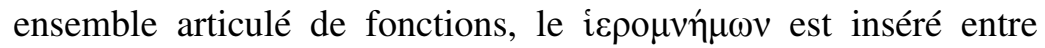

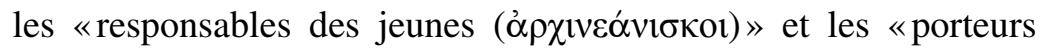
du feu ( $\pi \nu \rho \varphi o ́ \rho o u) »$. Le terme, peu répandu dans les associations religieuses, doit nécessairement comporter une connotation rituelle précise et désigner celui qui conserve et gère les formules liturgiques, tout en assumant des tâches financières/administratives ${ }^{41}$.

Une dernière inscription, un long texte sans doute contemporain (164-165 de notre ère), clôt le dossier épigraphique: le statut des Iobacchants $d$ 'Athènes ${ }^{42}$. Elle mentionne des dispositions administratives de l'association, des cotisations mensuelles et des amendes, des tâches des participants, etc., mais également, un trait intéressant du point de vue du rituel:

Que le prêtre accomplisse les services religieux traditionnels ( $\tau \dot{\alpha} \varsigma$

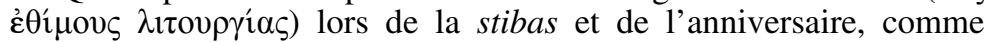
l'exigent les convenances, et qu'il donne la libation des Katagôgia, une seule pour la stibas, et qu'il prononce le sermon en l'honneur

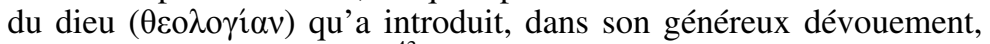
l'ancien prêtre Nikomakhos ${ }^{43}$.

Parmi les particularités de l'association apparaît l'introduction d'une $\theta \varepsilon 0 \lambda$ ới $\alpha$ (1. 115), un discours sur Dionysos déclamé lors des Katagôgia, le rituel qui célébrait le retour du dieu selon la réforme du prêtre Nikomakhos, dont la prêtrise date environ de 155 de notre ère ${ }^{44}$. Cette theologia portait davantage sur l'éloge du dieu que sur les aspects rituels de son culte ${ }^{45}$. Il reste difficile de savoir si le prêtre Nikomakhos a introduit l'usage de tenir un discours sur Dionysos lors des fêtes, ou bien s'il a fixé un discours qui était lu à l'occasion de ces fêtes. La différence n'est pas mince pour la compréhension

41. Cf. Robert Turcan, Liturgies, p. 69.

42. $S I G$ 1109, 111-117. Sur le texte du statut, Anne-Françoise Jaccottet, Choisir Dionysos, vol. II, p. 27-35 (n 4); plus généralement Luigi Moretti, «Il regolamento degli Iobacchi ateniesi», dans L'association dionysiaque, p. 247-259. La stibas était une fête annuelle de l'association des Iobacchants.

43. Traduction d'Anne-Françoise Jaccottet, Choisir Dionysos, vol. II, p. 33. Pour une réflexion sur la valeur de la stibas, Anne-Françoise Jaccottet, «Integrierte Andersartigkeit: Die Rolle der dionysischen Vereine», dans Renate Schlesier éd., A Different God? Dionysos and Ancient Polytheism, Berlin-Boston, 2011, p. 413-431.

44. Sur la datation de la prêtrise, Luigi Moretti, Il regolamento, p. 253. Sur les Katagôgies, Isabelle Tassignon, «Dionysos et les Katagôgies d'Asie Mineure», dans André Motte, Charles Ternes éds., Dieux, fêtes, sacré dans la Grèce et la Rome antiques, Turnhout, 2003, p. 80-99.

45. Cf. Henri Jeanmaire, Dionysos, p. 435 : «apparemment une composition roulant sur la légende ou la nature mystique du dieu». 
des pratiques dionysiaques, mais l'état fragmentaire et lacunaire des sources disponibles sur la nature et le contenu des logoi dédiés à Dionysos ne permet pas de trancher la question.

\section{ICONOGRAPHIE ET INITIATIONS DIONYSIAQUES}

Les traces et les indices de la présence de textesécrits dans les groupes initiatiques en l'honneur de Dionysos trouvent une confirmation dans l'analyse des représentations figurées, car ce n'est que la lecture croisée des sources qui permet de reconstruire le déroulement des teletai bachiques et de confirmer le rôle de l'écriture. Assurément l'analyse de l'iconographie exige de tenir compte du langage spécifique de ce type de documentation ${ }^{46}$ : les représentations figurées ne répondent pas nécessairement à des critères de vraisemblance et n'ont pas pour but d'illustrer le contenu et les protagonistes des pratiques rituelles. En outre, chaque image - comme chaque texte ou chaque inscription - doit être replacée dans son contexte d'origine, en considérant la fonction de l'espace qui l'entoure et les buts de sa présence dans un lieu. Ces précautions prises, on peut tenter une lecture des éléments matériels liés aux pratiques de l'écriture dans certaines représentations figurées dionysiaques.

Le cycle de fresques de la Villa des Mystères à Pompéi (70-60 avant notre ère), aussi célèbre que discuté, constitue un bon point de départ. La superbe mégalographie ornant le triclinium de la Villa a été diversement interprétée. Nous ne retiendrons que les deux principales propositions: selon les uns, les fresques représenteraient une scène de l'initiation dionysiaque, tandis que, pour d'autres, il n'y aurait pas de référence aux mystères dionysiaques, les images illustrant une scène interne au gynécée d'une matrone romaine à l'occasion de la célébration d'un mariage ${ }^{47}$. Sans entrer dans les détails

46. Sur le statut des images dans les pratiques rituelles des mondes anciens, Sylvia Estienne et al. éds., Image et religion dans l'antiquité gréco-romaine (Actes du Colloque de Rome, 11-13 décembre 2003, organisé par l'École française de Rome), Napoli, 2008. Plus spécialement sur l'art romain, Jaś Elsner, Art and the Roman Viewer: The Transformation of Art from Paganism to Christianity, Cambridge, 1995 et Jörg Rüpke, «Representation or Presence? Picturing the Divine in Ancient Rome», Archiv für Religionsgeschichte 12, 2010, p. 181-196.

47. La bibliographie à ce propos est considérable: pour la première hypothèse, Gilles Sauron, La grande fresque de la Villa des Mystères à Pompéi. Mémoires 
de la fresque, notons l'absence de preuve appuyant l'identification de cette salle comme lieu de culte d'initiés dionysiaques. Pourtant, s'il est vrai que les fresques ne sont pas une simple représentation d'une initiation dionysiaque (d'autres éléments figuratifs entrant en jeu), on ne peut nier que l'artiste (ou le commanditaire) a voulu évoquer des cérémonies dionysiaques de l'époque. Il ne s'agit pas d'une photographie des rituels, mais d'une représentation qui y fait allusion, comme dans les cas des sarcophages romains à représentations dionysiaques.

La scène qui se déroule sur la partie ouest de la paroi septentrionale présente un groupe de quatre personnes, dont les deux figures centrales sont une femme qui tient à la main un rouleau de papyrus et un enfant qui, à côté d'elle, est absorbé dans la lecture d'un autre rouleau ${ }^{48}$. La femme assise est interprétée généralement comme la figure préposée à l'enseignement dans la villa de Pompéi. À Rome, comme en Grèce, l'éducation religieuse des enfants - du moins dans la petite enfance ${ }^{49}$ - était assurée par les femmes de la maison. L'apprentissage renvoie également au rôle possible de la lecture d'un texte dans le cadre des pratiques et cérémonies dionysiaques, d'autant plus que les fresques de la maison sont parsemées d'autres références à des textes écrits, ce qui influe sur l'interprétation globale de la scène ${ }^{50}$. Prenons, par exemple, la figure féminine qui se trouve sur le mur occidental ${ }^{51}$. La femme représente la matrone, la domina de la maison: assise sur son fauteuil, le regard tourné vers le mur sud, elle observe le

d'une dévote de Dionysos, Paris, 1998 ; pour la deuxième, Paul Veyne, «La fresque dite des mystères à Pompéi», dans Paul Veyne, François Lissarrague, Françoise Frontisi-Ducroux, Les mystères du gynécée, Paris, 1998, p. 13-153.

48. L'image est accessible en ligne: http://commons.wikimedia.org/wiki/ File:Villa_dei_Misteri_II_-_2.jpg.

49. Sur l'éducation religieuse des enfants dans la Rome antique, bien qu'il n'y soit pas directement question de cette scène, Francesca Prescendi, «Children and the Transmission of Religious Knowledge», dans Véronique Dasen, Thomas Späth éds., Children, Memory, and Family Identity in Roman Culture, OxfordNew York, 2010, p. 73-94.

50. Ainsi, comme d'autres chercheurs, Gilles Sauron, La grande fresque, p. 115, interprète la scène comme une «lecture du rituel correspondant aux cérémonies dans la suite», alors que Paul Veyne, La fresque, p. 29-30, souligne qu'il ne s'agit pas d'une scène d'initiation et que la figure féminine ne peut être une esclave à cause de ses vêtements.

51. L'image est accessible en ligne: http://commons.wikimedia.org/wiki/ File:Villa_dei_Misteri_I_-_1.jpg. 
déroulement de la scène dans l'attente du rituel qui va avoir lieu. Sur l'accoudoir de gauche «est posé obliquement un diptyque avec son support $»^{52}$. Les tablettes sur lesquelles elle s'appuie sont vraisemblablement en relation avec le rite que les personnages de la scène sont en train de célébrer. Évidemment, comme pour toutes les figures et autres détails de la fresque, l'identification varie selon l'interprétation choisie: si la scène représente le gynécée lors d'un mariage, les tablettes deviennent le texte du contrat de mariage; mais elles sont un texte rituel pour ceux qui y reconnaissent une cérémonie dionysiaque ${ }^{53}$.

Un parallèle figuratif à cette image de Pompéi se trouve dans une mosaïque provenant d'Afrique du Nord, dans l'ensemble communément nommé «Maison de Dionysos» à Cuicul-Djemila (Algérie actuelle) qui date de la fin du $\mathrm{II}^{\mathrm{e}}$ ou du début du $\mathrm{III}^{\mathrm{e}}$ siècle [fig. 1]. Dans l'une des scènes composant le cadre de la mosaïque

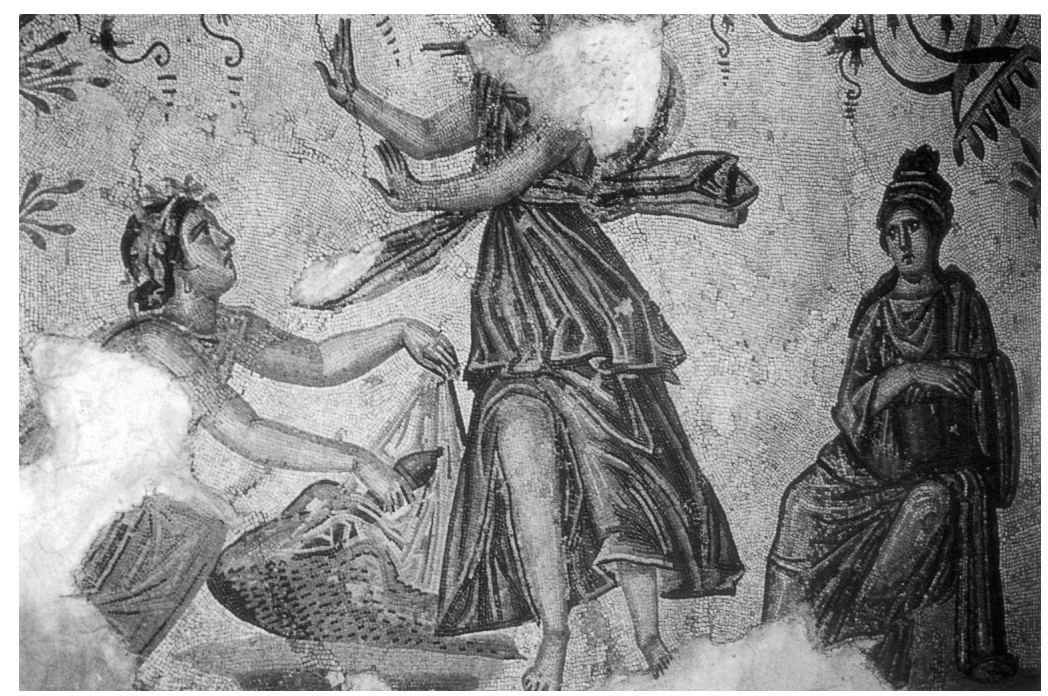

Fig. 1: Mosaïque de la «Maison de Dionysos», Djemila [Robert Turcan, Liturgies de l'initiation bacchique à l'époque romaine (LIBER). Documentation littéraire, inscrite et figurée, Paris, 2003, fig. 76]

52. Robert Turcan, Liturgies, p. 98.

53. Cf. Paul Veyne, La fresque, p. 32-37 et Gilles Sauron, La grande fresque, p. 76-77. 
(autour d'une représentation du mythe de Lycurgue, roi de Thrace, et d'Ambroisie) figure le dévoilement du phallos dionysiaque contenu à l'intérieur d'un liknon. Le phallos et le liknon sont deux éléments répandus dans les représentations iconographiques des initiations dionysiaques d'époque impériale, notamment sur les mosaïques et les sarcophages. À côté des deux figures qui dévoilent le phallos, une troisième se tient un peu à l'écart: elle a sur sa jambe un triptyque, sans doute en référence aux tablettes qui contenaient les prescriptions ou les enseignements nécessaires à l' accomplissement de l'initiation ou de la célébration dionysiaque ${ }^{54}$. La présence d'une tablette, ou plus généralement d'un texte écrit, est une manière d'évoquer visuellement (sans le décrire explicitement) le cadre rituel dionysiaque ${ }^{55}$.

Dans la Villa des Mystères, on remarque une autre référence à des textes écrits sur l'une des figures féminines qui préparent le déroulement du rituel ${ }^{56}$. La figure à gauche est en train de verser le contenu de son petit broc dans un vase lustral. À sa ceinture est inséré un rouleau de papyrus, qui pourrait être mis en relation avec la pratique rituelle en cours de déroulement: même si la plus grande prudence est de mise, la présence du papyrus pourrait, en effet, renvoyer au respect de l'ordo liturgique. Mais les références à la culture écrite ne s'arrêtent pas aux figures de la mégalographie. À côté de la salle décorée par la grande fresque, un cubiculum présente d'autres fresques à sujet dionysiaque moins étudiées: sept personnages bachiques (des satyres, des bacchantes et Dionysos) font écho à la scène de la grande salle ${ }^{57}$. On y voit entre autres une figure féminine couronnée, traditionnellement identifiée à la prêtresse du culte dionysiaque du fait de son costume typique des cérémonies bachiques; et elle tient à la main un rouleau. La présence de cet objet renvoie très probablement aux autres rouleaux présents dans la mégalographie de la salle adjacente. Il s'agit de comprendre le sens et le rôle du rouleau dans ces représentations

54. Cf. Robert Turcan, Liturgies, p. 126.

55. Sur la valeur initiatique de cette représentation iconographique, Fritz Graf, Sarah Iles Johnston, Ritual Texts, p. 148.

56. L'image est accessible en ligne: http://commons.wikimedia.org/wiki/ File:Villa-dei-misteri-pannel.jp.jpg?uselang=fr.

57. La scène du cubiculum 4 de la Villa des Mystères est analysée par Stéphanie Wyler, «Des images dionysiaques aux limites du religieux: le cubiculum 4 de la villa des Mystères », dans Sylvia Estienne et al. éds., Image et religion, p. 449-459. 
à sujet dionysiaque. Comme des études l'ont déjà montré, la représentation figurée de rouleaux ou de tablettes tenus en mains est fonctionnelle: elle cherche à attirer l'attention du spectateur sur la culture des personnages en question et, partant, sur l'expression de leur sagesse ${ }^{58}$. S'agit-il d'une culture littéraire ou bien d'un savoir religieux? Les deux interprétations ne s'excluent certes pas, mais il me semble que des rouleaux ou des tablettes associés à des figures accomplissant un rite, comme dans les cas de la Villa des Mystères à Pompéi ou de la mosaïque de Djemila, ne sauraient être étrangers à toute évocation de fonction ou valeur religieuse.

Une dernière référence iconographique, qui figure à nouveau des textes écrits dans des scènes d'initiations dionysiaques, provient de l'hypogée de la Porte Majeure à Rome, identifié comme «basilique pythagoricienne» depuis l'interprétation de Jérôme Carcopino en $1926^{59}$. Le bâtiment, dont la fonction est incertaine (tombe, nymphée, basilique funéraire, temple, etc.) date probablement de l'époque de Tibère ${ }^{60}$. Dans les stucs qui occupent la voûte apparaît la figure d'un jeune homme tenant à la main un objet qui ressemble à une grappe, flanqué d'une femme assise, tournée vers le jeune homme, un volumen à la main; sur la droite, une bacchante tient un thyrse alors qu'une autre figure féminine assise, plus complexe à interpréter, clôt la scène [fig. 2$]^{61}$. Une fois encore, dans un cadre que divers éléments connotent de façon dionysiaque, une figure féminine semble en relation avec un jeune homme par le biais de l'écriture, comme dans une relation de maître à élève: c'est ce que semble indiquer la position de la femme qui tient à la main le volumen, qui est la seule figure en position non frontale, et qui semble s'adresser au jeune homme. Doit-on lire, là également, une

58. Sur ce sujet, Guglielmo Cavallo, «Gli usi della cultura scritta nel mondo romano», dans Giovanni Pugliese Carratelli éd., Princeps Urbium. Cultura e vita sociale dell'Italia romana, Milano, 1991, p. 171-251, en particulier p. 221. Cf. aussi Anne Bielman, «Une vertu en rouleau ou comment la sagesse vint aux Grecques», dans Regula Frei Stolba et al. éds., Les femmes antiques entre sphère privée et sphère publique, Bern, 2003, p. 77-107.

59. Cf. Jérôme Carcopino, La basilique pythagoricienne de la Porte Majeure, Paris, 1926 et De Pythagore aux Apôtres. Études sur la conversion du monde romain, Paris, 1956, p. 9-23.

60. Pour une présentation de la basilique souterraine de la Porte Majeure et de son programme iconographique, Filippo Coarelli, Guide archéologique de Rome, Paris, 1994 [Roma-Bari, 1980], p. 161-164.

61. Pour la description de cette scène, Robert Turcan, Liturgies, p. 114. 
transmission d'éléments rituels ou narratifs en relation avec le rite, par le biais du support écrit? La proximité des schémas relevés jusqu'ici semble nous y autoriser.

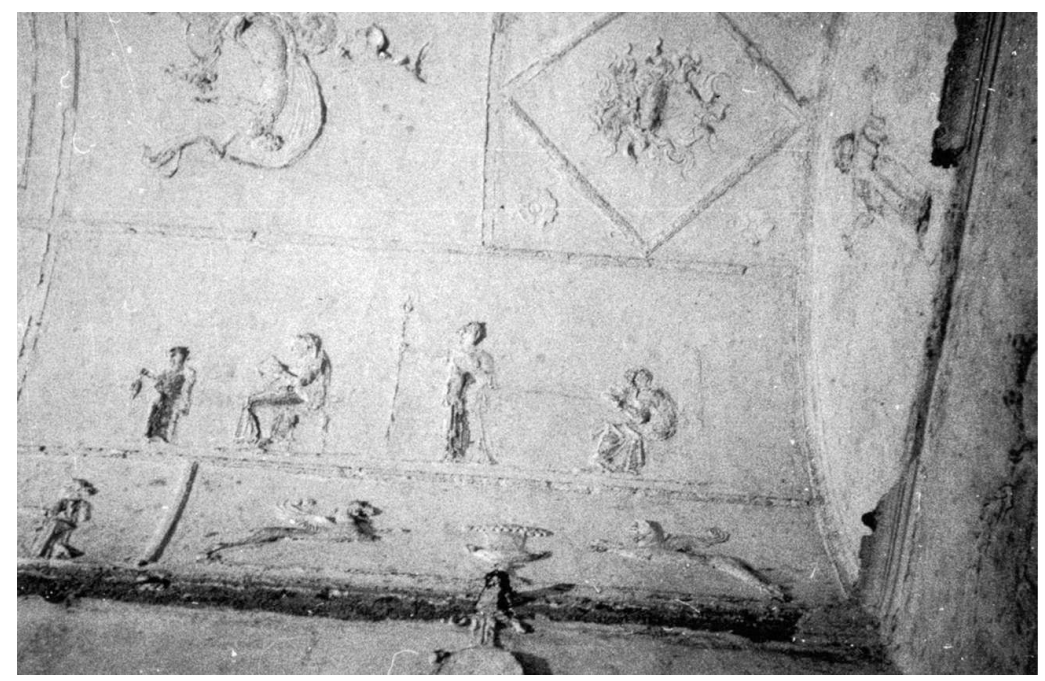

Fig. 2: Stuc de la voûte du bas côté droit de l'Hypogée de la Porte Majeure, Rome

[Robert Turcan, Liturgies de l'initiation bacchique à l'époque romaine (LIBER). Documentation littéraire, inscrite et figurée, Paris, 2003, fig. 47]

L'interprétation des scènes évoquées conduit à s'interroger sur un trait commun des témoignages iconographiques pris en compte. $\mathrm{Au}$ contraire des témoignages des inscriptions et des papyrus, les figures féminines tenant à la main des rouleaux de papyrus ou des tablettes sont récurrentes dans les représentations figurées et évoquent la présence de textes écrits dans le cadre des initiations dionysiaques. La raison de cette représentation au féminin est double. D'abord elle dérive de la référence mythique que les images sous-entendent: en effet, le jeune Dionysos - et les figures de la Villa des Mystères et de la Basilique de Porte Majeure sont aussi des jeunes - a été initié à ses propres cultes par une divinité féminine. Selon la version de la Bibliothèque du Pseudo-Apollodore (III, $5,1)$, Rhéa a révélé à Dionysos les connaissances indispensables pour accomplir les rituels; d'autres sources indiquent que la Mère 
des dieux lui a donné également les instruments nécessaires aux initiations ${ }^{62}$. Chez Nonnos de Panopolis, le rôle attribué à Rhéa chez Apollodore est confié à Mystis, personnification de l'initiation, qui enseigne les rites à mystères au Dionysos enfant et le prépare pour l'initiation $^{63}$; Nonnos attribue au dieu une fille, Teletè, dont le nom renvoie encore une fois aux initiations ${ }^{64}$. Ensuite, la prédominance féminine dérive du rôle que les femmes assumaient dans les groupes dionysiaques des époques hellénistique et impériale. Comme l'a clairement montré Anne-Françoise Jaccottet dans son étude sur les inscriptions des associations bachiques, bien que la structure hiérarchique des groupes dionysiaques reflète la structure de la société - en donnant aux hommes les fonctions les plus importantes -, les femmes assumaient une fonction spécifique dans la transmission des rituels et des pratiques cultuelles ${ }^{65}$. L'attribution de rouleaux ou de tablettes aux femmes dans les représentations figurées pourrait donc être issue de la fonction à la fois mythique et religieuse des «bacchantes» dans la pratique rituelle des époques hellénistique et romaine.

Les discussions savantes sur la valeur des images dans la reconstruction des pratiques rituelles dionysiaques sont particulièrement vives $^{66}$, notamment parce que ce type d'iconographie décorait souvent des espaces qui n'étaient pas réservés aux initiés, comme

62. Cf. Scholies à Lycophron 273.

63. Nonnos de Panopolis, Les Dionysiaques IX, 111-131.

64. Nonnos de Panopolis, Les Dionysiaques XVI, 400. La présence de Teletè dans le cadre des initiations dionysiaques est confirmée par les représentations des mosaïques, cf. Katherine M. D. Dunbabin, «Domestic Dionysus? Telete in Mosaics from Zeugma and the Late Roman Near East», Journal of Roman Archaeology 21, 2008, p. 193-224.

65. Anne-Françoise Jaccottet, Choisir Dionysos, vol. I, p. 71-100. Sur le rôle des femmes dans la transmission des savoirs dionysiaques, Francesco Massa, «Sacerdotesse, riformatrici ed educatrici : pratiche femminili nella trasmissione dei saperi dionisiaci», Storia delle Donne, 8, 2012, 105-126. L'article est accessible

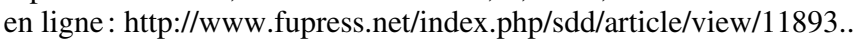

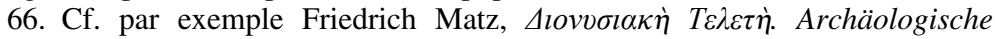
Untersuchungen zum Dionysoskult in hellenistischer und römischer, Mainz, 1963 , p. 7, qui soutenait que ce sont essentiellement les monuments qui donnent des informations sur l'initiation dionysiaque. Sur les sources figuratives, voir les réflexions de Robert Turcan, Liturgies, en particulier p. 135-141, et notamment d'Anne-Françoise Jaccottet, «Les mystères dionysiaques à l'époque romaine», Annuaire de l'EPHE, Section des Sciences Religieuses 114, 2005-2006, p. 235239. 
ces parties des habitations privées dédiées à l'accueil des hôtes ${ }^{67}$. La présence de ces images dans des contextes domestiques (ou funéraires, dans le cas des sarcophages) suggère une réflexion sur la nature et les buts auxquels les décorations dionysiaques se proposaient de répondre vis-à-vis de leurs spectateurs; mais surtout, comme l'a montré Stéphanie Wyler, «la pars priuata des villas, et en premier lieu les cubicula, sont le lieu privilégié de la revendication d'une culture personnelle, susceptibles de spéculations intellectuelles et de croyances, dont les images peuvent être les indices $»^{68}$. Dans ce contexte, malgré l'absence d'un effet de miroir ou d'une intention descriptive entre les images et la réalité cultuelle, pour que les représentations figurées soient compréhensibles aux yeux des spectateurs, elles devaient contenir des renvois plausibles aux pratiques rituelles. La comparaison entre textes, inscriptions et images nous invite à accepter un niveau de vraisemblance permettant aux "spectateurs" des représentations figurées de reconnaître certains éléments présents dans les occasions cultuelles, comme l'écrit et ses supports.

\section{Conclusions}

Le cadre qui émerge des sources dont nous disposons n'est pas homogène, car les témoignages n'ont pas la même importance dans toutes les zones géographiques. L'Égypte se présente comme le lieu de l'enregistrement des discours écrits dans un objectif à la fois politique et religieux. Cependant, bien que le texte sacré soit inscrit et enregistré dans les archives ptolémaïques, scellé et signé par celui qui le dépose, la mise par écrit ne cherche pas à répondre à la fixation ou à la création d'un canon. Hors de l'Égypte, les sources sont moins explicites, mais il est possible de tirer un certain nombre d'informations à partir des fonctions des associations dionysiaques et des références aux initiations bachiques dans les représentations

67. À ce propos, voir les travaux de Stéphanie Wyler: «Dionysos domesticus. Les motifs dionysiaques dans les maisons pompéiennes et romaines (II ${ }^{\mathrm{e}} \mathrm{s}$. av. $\mathrm{I}^{\mathrm{er}}$ s. ap. J.-C.)», Mélanges de l'École Française de Rome. Antiquité 116, 2004, p. 933-951, «Le dionysisme dans les villas romaines: initiation familiale ou contre-modèle social?», dans Perrine Galand-Hallyn, Carlos Levy éds., La villa et l'univers familial dans l'Antiquité et à la Renaissance, Paris, 2008, p. 61-77.

68. Stéphanie Wyler, «Des images dionysiaques», p. 459. 
iconographiques. Par delà les difficultés, la présence de textes écrits dans les pratiques initiatiques dionysiaques paraît être confirmée. Bien plus complexe se révèle la définition du contenu des textes intervenant dans un contexte rituel: les lamelles de Pelinna sont le seul témoignage qui nous permette de connaître explicitement les termes d'un texte initiatique dionysiaque. Mais le caractère spécifique de ces documents empêche de les considérer comme un exemple typique des écritures bachiques. Les rouleaux et tablettes relevés dans le parcours accompli jusqu'ici pouvaient contenir à la fois des références aux récits mythiques et des éléments utiles au déroulement des pratiques rituelles, comme l'illustre le Papyrus de Gurôb. La documentation en notre possession ne nous permet pas de lever complètement le voile et de répondre aux questions pourtant essentielles formulées en introduction.

Quoi qu'il en soit de leur contenu exact et de leur fonction, la mise par écrit des logoi dionysiaques tend à montrer la dimension variée des rituels et des croyances dionysiaques qui différaient souvent d'une région à l'autre, ou d'une association dionysiaque à l'autre ${ }^{69}$. L'usage de l'écriture s'affirme comme un support à la pluralité et à la variabilité des traditions mythiques et rituelles dionysiaques.

Nous évoquerons pour clore un texte plus tardif, le $D e$ errore profanarum religionum de Firmicus Maternus qui date de la première moitié du $\mathrm{IV}^{\mathrm{e}}$ siècle, après la mort de l'empereur Constantin. L'ouvrage témoigne de la mutation religieuse progressive de l'empire, et l'auteur en arrive même à encourager les nouveaux empereurs à éradiquer les cultes païens et à en détruire les temples ${ }^{70}$. Firmicus Maternus se focalise sur la reconstitution des cultes à mystères de l'époque tardive et sur une comparaison systématique entre les symbola de ces cultes et les citations des Écritures chrétiennes. L'auteur se propose explicitement d'attribuer au Diable l'origine des parallélismes entre rites païens et croyances chrétiennes, en exposant omnia symbola profanae religionis per ordinem. L'acclamation du dieu Dionysos comme dieu bicorne

69. À ce propos, Anne-Françoise Jaccottet, «Un dieu plusieurs mystères? Les différents visages des mystères dionysiaques», dans Corinne Bonnet, Jörg Rüpke, Paolo Scarpi éds., Religions orientales - culti misterici. Neue Perspektiven - nouvelles perspectives - prospettive nuove, Stuttgart, 2006, p. 219-230. $1-4$.

70. Firmicus Maternus, L'erreur des religions païennes XXVIII, 6 et XXIX, 
et l'utilisation cultuelle des serpents sont comparées au Livre d'Habacuc (III, 3-5) ou au Livre d'Isaïe (XXVII, 1) 11 , en justifiant le parallélisme entre les aspects traditionnels de Dionysos et les Écritures Saintes par l'action des démons ${ }^{72}$. Il est possible que certains de ces symbola dionysiaques soient contenus dans les textes sacrés des pratiques rituelles bachiques, car Firmicus Maternus a pu utiliser une source écrite pour en discuter dans son ouvrage. Ce qui est en jeu dans la deuxième partie du traité de Firmicus Maternus est une comparaison centrée sur les «écritures sacrées», une mise en évidence du conflit entre la seule et vraie Écriture Sainte et les formules des mystères qui ont essayé d'imiter les enseignements chrétiens sous l'influence du Diable et des démons. Le fait qu'au $I^{\mathrm{e}}$ siècle, dans l'esprit d'un auteur romain converti au christianisme, la comparaison entre croyances dionysiaques et croyances chrétiennes doive passer par la comparaison des textes écrits, signale le changement de statut de l'écriture dans son rapport avec les pratiques rituelles ${ }^{73}$.

f.massa@tin.it

71. Firmicus Maternus, L'erreur des religions païennes XXI, XXII et XXVI.

72. Cf. Francesco Massa, «In forma di serpente: incesti, mostri e diavoli nella condanna cristiana dei culti dionisiaci», dans Nicola Cusumano, Valeria Andò éds., Come bestie? Forme e paradossi della violenza tra mondo antico e disagio contemporaneo, Caltanisetta-Roma, 2010, p. 235-256.

73. Cf. Guy G. Stroumsa, La fin du sacrifice. Les mutations religieuses de l'Antiquité tardive, Paris, 2005, p. 63-101. - Le présent article doit beaucoup aux suggestions et aux remarques d'Anne-Françoise Jaccottet: qu'elle trouve ici l'expression de ma profonde gratitude. Bien entendu, les erreurs qui demeurent sont miennes. 